\title{
Yes-associated protein: A novel molecular target for the diagnosis, treatment and prognosis of hepatocellular carcinoma (Review)
}

\author{
LIANG SHAN $^{1}$, HONGYUAN JIANG ${ }^{1}$, LIFANG MA ${ }^{2}$ and YONGCHUN YU ${ }^{1}$ \\ Departments of ${ }^{1}$ Experiment Centre and ${ }^{2}$ Clinical Laboratory Medicine, Shanghai Municipal Hospital \\ of Traditional Chinese Medicine, Shanghai University of Traditional Chinese Medicine, Shanghai 200071, P.R. China
}

Received March 18, 2016; Accepted January 19, 2017

DOI: $10.3892 / \mathrm{ol} .2017 .6622$

\begin{abstract}
Hepatocellular carcinoma (HCC) is a common type of malignant tumor. The early-diagnosis and treatment options for HCC are limited, which is primarily due to an incomplete understanding of the underlying molecular mechanisms of the disease. Yes-associated protein (YAP) overexpression promotes proliferation and phenotypic transformation of HCC cells. Recently, elucidating the molecular mechanisms of the Hippo/YAP signaling pathway and investigating the interactions between the signaling molecules, as a potential strategy for the treatment of $\mathrm{HCC}$, has become an area of interest. The present review will discuss the role of YAP in HCC pathogenesis, and the significance of YAP in diagnosis, treatment and determining the prognosis.
\end{abstract}

\section{Contents}

1. Introduction

2. Overexpression of YAP promotes HCC

3. Significance of YAP in the diagnosis of $\mathrm{HCC}$

4. Role of YAP in the treatment of HCC

5. Role of YAP in determining the prognosis of $\mathrm{HCC}$

6. Conclusion

\section{Introduction}

The Hippo/Yes-associated protein (YAP) signaling pathway is a highly-conserved signal transduction pathway, which involves various oncogenes and tumor suppressor genes (1). Central to the signaling pathway is a core kinase cascade

Correspondence to: Professor Yongchun Yu, Department of Experiment Centre, Shanghai Municipal Hospital of Traditional Chinese Medicine, Shanghai University of Traditional Chinese Medicine, 274 Zhijiang Middle Road, Zhabei, Shanghai 200071, P.R. China

E-mail: yueyongchun88@163.com

Key words: yes-associated protein, Hippo signaling, oncoprotein, hepatocellular carcinoma, targeted treatment chain, which consists of mammalian STE20-like kinase 1/2 (MST1/2), human salvador 1 (hSAV1), large tumor suppressor kinase 1/2 (LATS1/2) and mps 1 binder kinase activator-like 1 (MOB1), and the downstream transcriptional coactivator YAP. Additionally, cell cycle protein $\mathrm{E}$ (cyclin E) and cellular inhibitor of apoptosis protein 1 (CIAP1) are regulatory factors downstream of the signaling pathway $(2,3)$.

Hippo/YAP signaling is a molecular switch for cell division and apoptosis, and YAP is the core component of the signaling pathway (4). Under physiological conditions, YAP is phosphorylated by proteins upstream of the Hippo/YAP signaling pathway via a series of kinase cascades $(5,6)$. Initially, MST1/2 binds to hSAV1, which forms a complex and phosphorylates LATS1/2. YAP is subsequently phosphorylated by LATS1/2, which is regulated by MOB1. Phosphorylation of YAP subsequently promotes cytoplasmic localization of the YAP [serine (ser) 127]/14-3-3 protein complex. This in turn prevents YAP-mediated transcriptional activation in the nucleus $(5,6)$ (Fig. 1).

Additionally, YAP has a WW domain that is able to directly associate with PPxY motifs in LATS1/2 for signal transduction. This suggests that Hippo/YAP signaling is also able to inhibit YAP by non-phosphorylation (7). When Hippo/YAP signaling is aberrant, overexpressed YAP enters into the nucleus and activates the transcription factor TEA domain family member (TEAD), which in turn stimulates the transcription of downstream factors cyclin E, CIAP1 and connective tissue growth factor (CTGF). Therefore, YAP is able to promote proliferation and inhibit apoptosis (8). Notably, the WW domain of YAP is also able to promote apoptosis following DNA damage via binding to $\mathrm{p} 73$, which may be associated with the microenvironment or type of tumor (9).

Hepatocellular carcinoma (HCC) is a common type of malignant tumor and the third leading cause of cancer-associated mortality worldwide (10). HCC is induced by numerous factors and develops in multiple stages, which hinders early detection. YAP is able to promote HCC cell proliferation and transformation (a change in cell phenotype) $(11,12)$. Therefore, investigating the association between YAP and $\mathrm{HCC}$ is important for identifying novel tumor markers. YAP-associated membrane proteins, including cluster of differentiation (CD)166 and melanoma cell adhesion molecule (MCAM), have been reported to be involved in hepatocarcinogenesis (13). 
Additionally, a number of studies have demonstrated that the targeting of Hippo/YAP signaling may be able to inhibit proliferation in HCC cells $(14,15)$. Studies have employed two main strategies: i) Targeting components of the Hippo/YAP signaling pathway, including inhibiting the activity of YAP or promoting the activity of MST1 or LATS1/2 (16-18); and ii) targeting pathways and factors associated with the Hippo/YAP signaling pathway, including Wnt/ß-catenin, cyclic adenosine monophosphate response element-binding protein (CREB) and tribbles homolog 2 (TRIB2) $(12,19,20)$.

YAP may also be used to determine the prognosis of HCC. The observation that liver regeneration stimulates the growth of residual cancer tissue following surgery has been demonstrated by multiple clinical studies. However, the precise underlying mechanisms remain unclear (21). Furthermore, YAP is important for regulating liver size, and YAP-associated HCC is linked with a poor short-term prognosis, suggesting that YAP may be utilized as an independent prognostic marker for HCC (22). In conclusion, YAP exerts an important role in the development of HCC. Further elucidation of the underlying molecular mechanisms of the Hippo/YAP signaling pathway and the interaction between different signaling pathways and molecules may provide novel methods for the diagnosis, treatment and prognosis of HCC.

In the present review, the underlying molecular mechanisms of Hippo/YAP signaling and YAP-associated proteins in HCC pathogenesis were examined. In addition, the corresponding advantages of YAP in diagnosis, treatment and determining the prognosis of $\mathrm{HCC}$ were analyzed.

\section{Overexpression of YAP promotes HCC}

HCC is a highly malignant and aggressive cancer, and YAP is overexpressed in $62 \%$ of patients with HCC (23). The overexpression of YAP has been reported in rat and human HCC cells (24). Another study demonstrated that YAP is present in the HCC cell nuclei during hepatocarcinogenesis (25). Furthermore, the expression of YAP has also been associated with the differentiation of HCC cells, level of serum $\alpha$-fetoprotein (AFP) and vascular infiltration $(26,27)$.

The activation of hepatic stellate cells (HSCs) has been demonstrated to be critical for the development of cirrhosis from hepatic fibrosis, and that it promotes the proliferation and survival of HCC cells (28). It has been reported that a complex loop of regulation between HSCs and YAP is involved in hepatocarcinogenesis. Initially, the overexpression of YAP promotes the HCC cells to secrete more CTGF, which in turn regulates HSC function. The extracellular matrix (ECM) is subsequently activated by HSCs. As a result of the increasing activity of ECM in the HCC microenvironment, YAP is translocated from the cytoplasm into the nucleus, and YAP-mediated gene transcription is activated (29-31). Notably, the knockdown of YAP has been observed to inhibit the pluripotency of embryonic stem (ES) cells. By contrast, overexpression of YAP inhibits ES cell differentiation (32). These studies suggest that the overexpression of YAP promotes HCC cell proliferation and transformation (change in cell phenotype), and that YAP may have a critical role in the development of hepatocarcinogenesis.

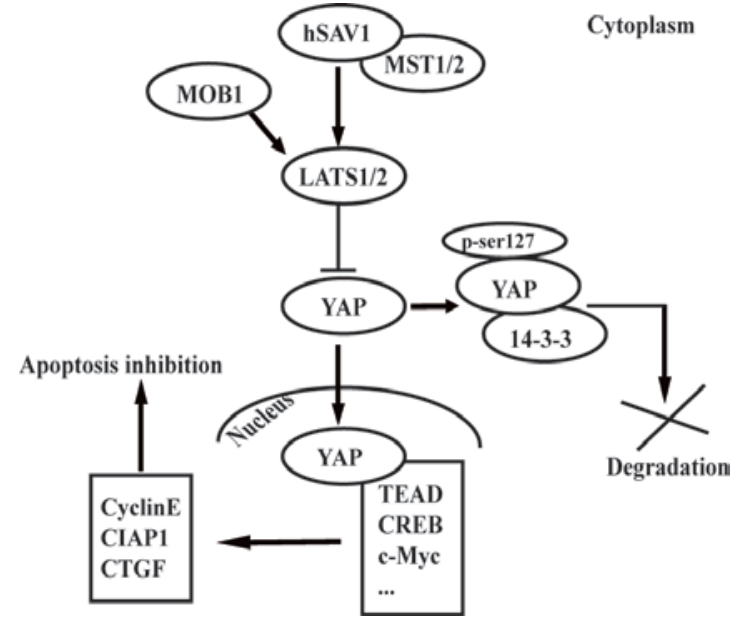

Figure 1. Hippo/YAP signaling pathway maintains the balance between cell proliferation and apoptosis. YAP is a key factor downstream of the signaling pathway. Under physiological conditions, YAP is phosphorylated through a series of kinase cascades and is located in the cytoplasm. When MST1/2, hSAV1, MOB1 and LATS1/2 are inactivated or YAP is overexpressed, YAP is translocated into the nucleus and binds to TEAD. This in turn promotes the expression of a number of factors (cyclin E, CIAP1 and CTGF). This also promotes cell proliferation and inhibits apoptosis. CIAP1, cellular inhibitor of apoptosis protein 1; CREB, cyclic adenosine monophosphate response element-binding protein; CTGF, connective tissue growth factor; hSAV1, human salvador 1 ; LATS1/2, large tumor suppressor kinase $1 / 2$; MOB1, mps 1 binder kinase activator-like 1; MST1/2, mammalian STE20-like kinase 1/2; se, serine; TEAD, TEA domain family member; YAP, yes-associated protein.

\section{Significance of YAP in the diagnosis of HCC}

Tumor screening tests require markers with high sensitivity and specificity. YAP is overexpressed in patients with $\mathrm{HCC}$, which suggests that it can be used as a clinical tumor marker (23).

A previous study suggested that the joint detection of YAP and AFP is specific for HCC, and that this joint detection may improve the diagnosis rate (21). However, YAP is predominantly located in the nuclei of HCC cells, which makes detection difficult for diagnosis. By contrast, membrane proteins involved in the organization of tumor cells are readily released into the blood. Therefore, utilizing YAP-associated membrane proteins to aid the clinical diagnosis of $\mathrm{HCC}$ has become an area of interest.

The membrane proteins CD166 and MCAM are potential candidates for HCC diagnostic markers. CD166 is an upstream regulatory factor of YAP, and it is also upregulated in HCC cells. CD166 promotes YAP function in hepatocarcinogenesis (33). MCAM is a specific target of YAP in HCC, and MCAM expression is upregulated in HCC cells. It has been reported that the serum levels of MCAM and AFP are positively correlated (34).

In conclusion, serum CD166 and MCAM may replace YAP as tumor markers for YAP-associated HCC. The elucidation of the molecular mechanisms of YAP and YAP-associated membrane proteins may aid the development of non-invasive tests for the diagnosis of HCC.

\section{Role of YAP in the treatment of HCC}

The Hippo/YAP signaling pathway involves a number of oncogenes and tumor suppressor genes. The inactivation 
of this signaling pathway leads to an imbalance between cell proliferation and apoptosis, which induces hepatocarcinogenesis (35). Investigating the molecular mechanisms of the Hippo/YAP pathway and other associated factors is beneficial for elucidating the novel targets for the treatment of HCC.

Use of Hippo/YAP signaling molecules as targets for the treatment of HCC. YAP is an oncogene. Inhibiting the activity of YAP may be beneficial for the treatment of HCC (36). Harvey et al (36) proposed that YAP1 may be a potential target for tumor treatment. However, it was suggested that although therapies that target YAP1 would be specific, drug resistance is likely to occur (36). Studies have demonstrated that knocking down YAP via short hairpin RNAs and small interfering RNAs can lead to a decrease in the activity of cyclin E, and reduce cell proliferation and adhesion $(24,37)$. Furthermore, it has been observed that phosphorylation of YAP (ser127) is promoted by overexpression of MST1 (38). This suggests that MST1 overexpression is able to inhibit hepatocarcinogenesis, therefore, it may be a potential target for the treatment of HCC (38).

A number of microRNAs (miRs/miRNAs) have also been implicated in hepatocarcinogenesis. However, the interactions between YAP and miRNAs remain unclear. A previous study demonstrated that the ectopic expression of miR-375 decreases the transcriptional activity of YAP mRNA and inhibits the expression of endogenous YAP proteins (39). Additionally, Wang et al (40) demonstrated that miR-506 inhibits YAP expression by targeting the 3'-untranslated region of YAP mRNA and reduces the proliferation of HCC cells. This indicates that utilizing miR-375 or miR-506 to target YAP may be a potential strategy to inhibit the proliferation of HCC cells.

YAP may be targeted to increase the sensitivity of tumor cells to chemotherapy. It has been reported that the expression of YAP mRNA increases when HepG2 cells are treated with doxorubicin $(0.7 \mu \mathrm{g} / \mathrm{ml})$, and that the chemosensitivity of the cells increases when expression of YAP is increased $(41,42)$. It has been observed that during chemotherapy, p53 is able to promote the expression of YAP by binding to the YAP gene promoter in the 2,300 to $2,105 \mathrm{bp}$ region $(41,42)$. These studies suggest that increasing the expression levels of the regulatory factors of the Hippo pathway and reducing the expression of YAP may be a method for treating HCC. Understanding the precise mechanisms of these interactions would assist in the development of novel sensitizers for chemotherapy.

Use of YAP-independent factors as targets for the treatment of HCC. Hepatocarcinogenesis is regulated by numerous factors (43). YAP expression is not only regulated by the Hippo/YAP signaling pathway, but is associated with the activation of YAP-dependent factors; therefore, the Hippo/YAP signaling pathway may not be the only target for the treatment of HCC (33). The main mechanisms between YAP and YAP-independent transcription factors are presented in Table I.

CREB promotes the transcription of YAP by binding to its promoter (2,608 to 2,439 bp). YAP inhibits CREB degradation following phosphorylation by interacting with mitogen-activated protein kinase 14 and $\beta$-transducin repeat-containing
E3 ubiquitin protein ligase (BTRC). The interaction between CREB and YAP is important for HCC cell survival and change in cell phenotype $(12,44)$.

The Wnt signaling pathway regulates cell proliferation, differentiation and activity. Studies have reported that phosphorylation of YAP inhibits Wnt/ $\beta$-catenin signaling (19). Additionally, other studies have reported that TRIB2 is a direct target of Wnt/T-cell factor signaling in HCC. It has also been demonstrated that TRIB2 is able to inhibit BTRC, which reduces CCAAT/enhancer binding protein $\alpha$ $(\mathrm{C} / \mathrm{EBP} \alpha)$-mediated inhibition of the transcriptional activity of YAP/TEAD. These studies suggest that TRIB2 may be a key modulator with Wnt/ $\beta$-catenin, Hippo/YAP and $\mathrm{C} / \mathrm{EBP} \alpha$ signaling; therefore, TRIB2 may be another target for the treatment of HCC $(20,45,46)$.

c-Myc may be involved in regulating the transcriptional activity of YAP. Xiao et al (47) demonstrated that YAP-knockdown inhibits the expression of c-Myc. Further investigation indicated that although c-Myc-knockdown has no effect on YAP mRNA levels, it inhibits the expression of human telomerase reverse transcriptase, which is a downstream target of YAP.

The interactions between Hippo/YAP signaling and a number of factors, including CD44 (48), Sonic hedgehog (49), Notch (50), insulin-like growth factor 1 receptor/protein kinase B (AKT) (51) and phosphatidylinositol 3-kinase/AKT (52), are also important in hepatocarcinogenesis and represent potential therapeutic targets for HCC. In conclusion, hepatocarcinogenesis is a complex process, and understanding the interactions between regulatory signals is useful for altering Hippo/YAP signaling and for identifying novel targets for the treatment of HCC (Fig. 2).

\section{Role of YAP in determining the prognosis of $\mathrm{HCC}$}

Currently, surgical resection is the preferred treatment for HCC. However, the 5-year survival rate is only 20 to $40 \%$ (53). Following surgery, residual cancer tissues are highly proliferative, and overexpression of YAP accelerates the proliferation of HCC cells. Expression of YAP, a tumor diameter of $>5 \mathrm{~cm}$, poor differentiation and AJCC stage III are independent risk factors of tumor recurrence post-surgery (4). Consequently, it is important to investigate the molecular mechanisms of YAP signaling and its effect in determining the prognosis of HCC.

$\mathrm{Xu}$ et al (23) analyzed the clinical and pathological data of 166 patients with HCC and observed that the overexpression of YAP in HCC cells is associated with a short disease-free survival (DFS) time (log-rank=3.252; $\mathrm{P}=0.071$ ) using Kaplan-Meier analysis. The DFS time was 27.1 months [95\% confidence interval (CI), 19.5-34.7] for the YAP-negative group (n=64) and 14.5 months (95\% CI, 10.8-18.2) for the YAP-positive group $(n=102)$. Therefore, the survival time in HCC patients with YAP overexpression was significantly reduced compared with that in patients with YAP-negative HCC following surgery (23). These findings demonstrated that there is early cancer recurrence and a poorer prognosis in YAP-associated HCC, suggesting that altering the expression of YAP post-surgery may be useful to prolong the survival time of patients. 
Table I. Interaction between YAP and YAP-independent transcription factors in HCC.

Transcription factor Mechanism

(Refs.)

TEAD

Dephosphorylated YAP binds to TEAD via its WW domain, which promotes cell

CD166 CD166 modulates the activity of YAP at the transcriptional and post-transcriptional proliferation and inhibits apoptosis. levels. YAP is regulated transcriptionally by CD166 through its interaction with CREB and post-transcriptionally by CD166-mediated inhibition of AMOT130, which affects the stability of YAP.

MCAM High levels of p300 promote the binding of YAP to the MCAM promoter, which subsequently promotes histone acetylation and polymerase II recruitment through the dissociation of the deacetylase Sirt1.

CREB YAP inhibits the degradation of CREB, which is mediated by BTRC and p38.

The accumulation of CREB stimulates transcription of YAP.

TRIB2 TRIB2 represses the $\mathrm{C} / \mathrm{EBP} \alpha$-mediated inhibition of YAP/TEAD transcriptional activation in HCC cells.

c-Myc YAP promotes c-Myc transcriptional output via c-Abl. c-Myc promotes protein expression independent of transcription.

Shh

Shh promotes YAP translocation into the nucleus. Shh stabilizes IRS1, and the interactions between IRS 1 and YAP determine the subcellular localization of YAP.

BTRC, $\beta$-transducin repeat-containing E3 ubiquitin protein ligase; TEAD, TEA domain family member; CD, cluster of differentiation; $\mathrm{C} / \mathrm{EBP} \alpha, \mathrm{CCAAT} /$ enhancer binding protein $\alpha$; MCAM, melanoma cell adhesion molecule; CREB, cyclic adenosine monophosphate response element-binding protein; TRIB2, tribbles homolog 2; IRS1, insulin receptor substrate 1; Shh, Sonic hedgehog protein; YAP, yes-associated protein.

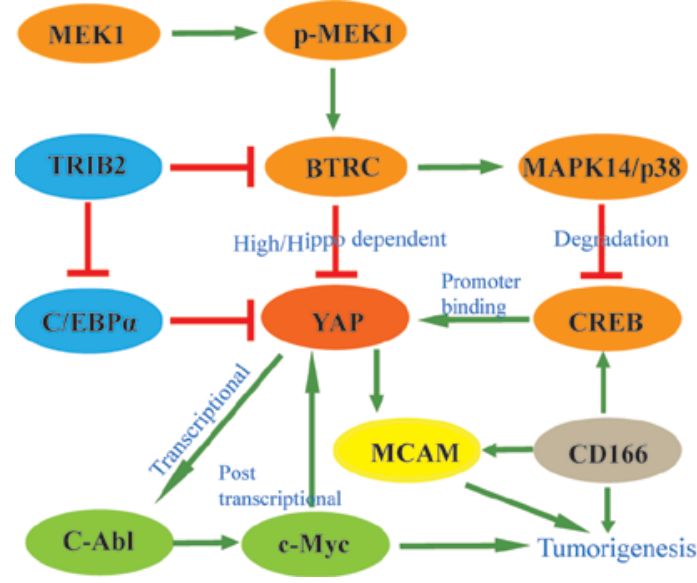

Figure 2. YAP is regulated by various factors during hepatocarcinogenesis in addition to the members of the Hippo signaling pathway. These factors have an important role in YAP-associated hepatocellular carcinoma. BTRC and TRIB2 inhibit the overexpression of YAP. The interactions between YAP and other factors (CREB, c-Myc, CD166 and MCAM) promote hepatocarcinogenesis. High/hippo dependent refers to the effects of BTRC on YAP, which are dependent on the Hippo pathway. When BTRC is overexpressed, or the Hippo pathway is activated and YAP turnover is inhibited; however, at normal levels of BTRC, YAP is protected from degradation. $\mathrm{BTRC}, \beta$-transducin repeat-containing E3 ubiquitin protein ligase; $\mathrm{CD}$, cluster of differentiation; $\mathrm{CREB}$, cyclic adenosine monophosphate response element-binding protein; $\mathrm{C} / \mathrm{EBP} \alpha, \mathrm{CCAAT} / \mathrm{enh}$ ancer binding protein $\alpha$; MAPK14, mitogen-activated protein kinase 14; MCAM, melanoma cell adhesion molecule; p-MEK1, phosphorylated-mitogen-activated protein kinase; TRIB2, tribbles homolog 2; YAP, yes-associated protein.

Furthermore, F-box and WD repeat domain-containing 7 (Fbxw7), an E3 ubiquitin ligase, may be a potential independent prognostic marker for YAP-associated HCC. A previous study demonstrated that $\mathrm{Fbxw} 7$ expression inhibits proliferation and induces apoptosis in HCC cells by promoting the ubiquitination and proteasomal degradation of YAP, which is crucial for improving the 5-year survival rate (54).

\section{Conclusion}

Compared with conventional therapies (radiotherapy and chemotherapy), targeting YAP and YAP-associated proteins for the treatment of HCC has the advantage of high selectivity and specificity (16). YAP and associated membrane proteins, which are specifically overexpressed in HCC, may be used as markers to aid in the early diagnosis of HCC.

The molecular mechanisms of Hippo/YAP signaling and YAP-associated proteins have been gradually elucidated. The factors identified include YAP, MST1/2, CREB, TRIB2, c-Myc, miR-506, CD44, Sonic hedgehog, Notch, insulin-like growth factor 1 receptor/AKT, phosphatidylinositol 3-kinase/AKT and Wnt/ $\beta$-catenin, which have been implicated in HCC differentiation, the cell cycle, apoptosis, migration, invasion, and lymph node and systemic metastasis. Therefore, investigations into the underlying molecular mechanisms of interaction between these pathways are beneficial for the development of high-efficiency and broad-spectrum targeting drugs.

YAP may be employed as an independent prognostic marker for HCC. YAP-associated HCC is highly malignant and aggressive. The recovery rate is low and the prognosis is poor. Overexpression of YAP during liver regeneration is a leading cause of early cancer recurrence. This suggests that detecting the change in YAP expression post-surgery may 
be beneficial for the early detection of HCC recurrence and metastasis.

In conclusion, the overexpression of YAP promotes cell proliferation and phenotypic change in HCC cells. The interactions between Hippo/YAP signaling and its associated-proteins, which are involved in hepatocarcinogenesis, constitute a complex network. The components in this network are crucial for HCC diagnosis, treatment and prognosis.

The development of HCC treatments targeting YAP may be a long process. Correlative studies have made considerable progress. However since the exact molecular mechanisms underlying the pathogenesis of HCC are not fully understood, the identification of specific proteins as therapeutic targets for treatment has considerable limitations. Therefore, elucidating how YAP is dysregulated in HCC will be pivotal for determining the factors that should be targeted. Resolving the problem of primary and acquired drug resistance during YAP-targeting therapy is also a challenge. Notably, a number of studies have reported that YAP is able to act as an oncogene and a tumor suppressor gene, indicating that HCC treatments targeting YAP require further investigation. In conclusion, whilst the development of therapies involving the targeting of Hippo/YAP signaling is at its infancy, these therapies may be novel strategies for treating HCC.

\section{References}

1. Huang J, Wu S, Barrera J, Matthews K and Pan D: The Hippo signaling pathway coordinately regulates cell proliferation and apoptosis by inactivating Yorkie, the Drosophila homolog of YAP. Cell 122: 421-434, 2005.

2. Hilman D and Gat U: The evolutionary history of YAP and the Hippo/YAP pathway. Mol Biol Evol 28: 2403-2417, 2011.

3. Yu FX and Guan KL: The Hippo pathway: Regulators and regulations. Genes Dev 27: 355-371, 2013.

4. Zeng Q and Hong W: The emerging role of the Hippo pathway in cell contact inhibition, organ size control and cancer development in mammals. Cancer Cell 13: 188-192, 2008.

5. Halder G and Johnson RL: Hippo signaling: Growth control and beyond. Development 138: 9-22, 2011.

6. Oh $\mathrm{H}$ and Irvine KD: Yorkie: The final destination of Hippo signaling. Trends Cell Biol 20: 410-417, 2010.

7. Schlegelmilch K, Mohseni M, Kirak O, Pruszak J, Rodriguez JR, Zhou D, Kreger BT, Vasioukhin V, Avruch J, Brummelkamp TR and Camargo FD: YAP1 acts downstream of $\alpha$-catenin to control epidermal proliferation. Cell 144: 782-795, 2011.

8. Zhang J, Ji JY, Yu M, Overholtzer M, Smolen GA, Wang R, Brugge JS, Dyson NJ and Haber DA: YAP-dependent induction of amphiregulin identifies a non-cell-autonomous component of the Hippo pathway. Nat Cell Biol 11: 1444-1450, 2009.

9. Strano S and Blandino G: YAP1 meets tumor suppression. Mol Cell 27: 863-864, 2007.

10. Farazi PA and Depinho RA: Hepatocellular carcinoma pathogenesis: From genes to environment. Nat Rev Cancer 6: 674-687, 2006.

11. Dong J, Feldmann G, Huang J, Wu S, Zhang N, Comerford SA, Gayyed MF, Anders RA, Maitra A and Pan D: Elucidation of a universal size-control mechanism in drosophila and mammals. Cell 130: 1120-1133, 2007.

12. Wang J, Ma L, Weng W, Qiao Y, Zhang Y, He J, Wang H, Xiao W, Li L, Chu Q, et al: Mutual interaction between YAP and CREB promotes tumorigenesis in liver cancer. Hepatology 58: 1011-1020, 2013.

13. Tang X, Chen X, Xu Y, Qiao Y, Zhang X, Wang Y, Guan Y, Sun F and Wang J: CD166 positively regulates MCAM via inhibition to ubiquitin E3 ligases Smurf1 and $\beta$ TrCP through PI3K/AKT and c-Raf/MEK/ERK signaling in Bel-7402 hepatocellular carcinoma cells. Cell Signal 27: 1694-1702, 2015.

14. Wang Y, Fang R, Cui M, Zhang W, Bai X, Wang H, Liu B, Zhang $X$ and Ye L: The oncoprotein HBXIP up-regulates YAP through activation of transcription factor c-Myb to promote growth of liver cancer. Cancer Lett 385: 234-242, 2017.
15. Xu G, Wang J, Wu F, Wang N, Zhou W, Wang Q, Pan W, Ao G and Yang J: YAP and 14-3-3 $\gamma$ are involved in HS-OA-induced growth inhibition of hepatocellular carcinoma cells: A novel mechanism for hydrogen sulfide releasing oleanolic acid. Oncotarget 7: 52150-52165, 2016.

16. Perra A, Kowalik MA, Ghiso E, Ledda-Columbano GM, Di Tommaso L, Angioni MM, Raschioni C, Testore E, Roncalli M, Giordano S and Columbano A: YAP activation is an early event and a potential therapeutic target in liver cancer development. J Hepatol 61: 1088-1096, 2014.

17. Loforese G, Malinka T, Keogh A, Baier F, Simillion C, Montani M, Halazonetis TD, Candinas D and Stroka D: Impaired liver regeneration in aged mice can be rescued by silencing Hippo core kinases MST1 and MST2. EMBO Mol Med 9: 46-60, 2017.

18. Yi J, Lu L, Yanger K, Wang W, Sohn BH, Stanger BZ, Zhang M, Martin JF, Ajani JA, Chen J, et al: Large tumor suppressor homo$\operatorname{logs} 1$ and 2 regulate mouse liver progenitor cell proliferation and maturation through antagonism of the coactivators YAP and TAZ. Hepatology 64: 1757-1772, 2016.

19. Perumal N, Perumal M, Kannan A, Subramani K, Halagowder D and Sivasithamparam N: Morin impedes Yap nuclear translocation and fosters apoptosis through suppression of Wnt/ $\beta$-catenin and NF- $\mathrm{BB}$ signaling in Mst1 overexpressed HepG2 cells. Exp Cell Res 355: 124-141, 2017.

20. Wang J, Park J, Wei Y, Rajurkar M, Cotton JL, Fan Q, Lewis BC, Ji $\mathrm{H}$ and Mao J: TRIB2 acts downstream of Wnt/TCF in liver cancer cells to regulate YAP and $\mathrm{C} / \mathrm{EBP} \alpha$ function. Mol Cell 51: 211-225, 2013.

21. Wang YP and Tang DX: Expression of Yes-associated protein in liver cancer and its correlation with clinicopathological features and prognosis of liver cancer patients. Int J Clin Exp Med 8: 1080-1086, 2015.

22. Tordjmann T: Hippo signaling: Liver size regulation and beyond. Clin Res Hepatol Gastroenterol 35: 344-346, 2011.

23. Xu MZ, Yao TJ, Lee NP, Ng IO, Chan YT, Zender L, Lowe SW, Poon RT and Luk JM: Yes-associated protein is an independent prognostic marker in hepatocellular carcinoma. Cancer 115: 4576-4585, 2009.

24. Zender L, Spector MS, Xue W, Flemming P, Cordon-Cardo C, Silke J, Fan ST, Luk JM, Wigler M, Hannon GJ, et al: Idetification and validation of oncogenes in liver cancer using an integrative oncogenomic approach. Cell 125: 1253-1267, 2006.

25. Zhao B, Ye X, Yu J, Li L, Li W, Li S, Yu J, Lin JD, Wang CY, Chinnaiyan AM, et al: TEAD mediates YAP-dependent gene induction and growth control. Genes Dev 22: 1962-1971, 2008.

26. Cai WY, Lin LY, Hao H, Zhang SM, Ma F, Hong XX, Zhang H, Liu QF, Ye GD, Sun GB, et al: Yes-associated protein/TEA domain family member and hepatocyte nuclear factor 4-alpha $(\mathrm{HNF} 4 \alpha)$ repress reciprocally to regulate hepatocarcinogenesis in rats and mice. Hepatology 65: 1206-1221, 2017.

27. Lin C, Hu Z, Lei B, Tang B, Yu H, Qiu X and He S: Overexpression of Yes-associated protein and its association with clinicopathological features of hepatocellular carcinoma: A meta-analysis. Liver Int: Mar 26, 2017 (Epub ahead of print).

28. Song Y, Kim SH, Kim KM, Choi EK, Kim J and Seo HR: Activated hepatic stellate cells play pivotal roles in hepatocellular carcinoma cell chemoresistance and migration in multicellular tumor spheroids. Sci Rep 6: 36750, 2016.

29. Mannaerts I, Leite SB, Verhulst S, Claerhout S, Eysackers N, Thoen LF, Hoorens A, Reynaert H, Halder G and van Grunsven LA: The Hippo pathway effector YAP controls mouse hepatic stellate cell activation. J Hepatol 63: 679-688, 2015.

30. Paget S: The distribution of secondary growths in cancer of the breast. 1989. Cancer Metastasis Rev 8: 98-101, 1989.

31. Okabe H, Beppu T, Hayashi H, Horino K, Masuda T, Komori H, Ishikawa $\mathrm{S}$, Watanabe $\mathrm{M}$, Takamori $\mathrm{H}$, Iyama $\mathrm{K}$ and Baba $\mathrm{H}$ : Hepatic stellate cells may relate to progression of intrahepatic cholangiocarcinoma. Ann Surg Oncol 16: 2555-2564, 2009.

32. Lian I, Kim J, Okazawa H, Zhao J, Zhao B, Yu J, Chinnaiyan A, Israel MA, Goldstein LS, Abujarour R, et al: The role of YAP transcription coactivator in regulating stem cell self-renewal and differentiation. Genes Dev 24: 1106-1118, 2010.

33. Ma L, Lin J, Qiao Y, Weng W, Liu W, Wang J and Sun F: Serum CD166: A novel hepatocellular carcinoma tumor marker. Clin Chim Acta 441: 156-162, 2015.

34. Wang J, Tang X, Weng W, Qiao Y, Lin J, Liu W, Liu R, Ma L, Yu W, Yu Y, et al: The membrane protein melanoma cell adhesion molecule (MCAM) is a novel tumor marker that stimulates tumorigenesis in hepatocellular carcinoma. Oncogene 36: 5781-5795, 2015. 
35. Camargo FD, Gokhale S, Johnnidis JB, Fu D, Bell GW, Jaenisch R and Brummelkamp TR: YAP1 increases organ size and expands undifferentiated progenitor cells. Curr Biol 17: 2054-2060, 2007.

36. Harvey KF, Zhang X and Thomas DM: The Hippo pathway and human cancer. Nat Rev Cancer 13: 246-257, 2013.

37. Liu AM, Xu MZ, Chen J, Poon RT and Luk JM: Targeting YAP and Hippo signaling pathway in liver cancer. Expert Opin Ther Targets 14: 855-868, 2010.

38. Zhou D, Conrad C, Xia F, Park JS, Payer B, Yin Y, Lauwers GY, Thasler W, Lee JT, Avruch J and Bardeesy N: Mst1 and Mst2 maintain hepatocyte quiescence and sup- press hepatocellular carcinoma development through inac-tivation of the Yap1 oncogene. Cancer Cell 16: 425-438, 2009.

39. Liu AM, Poon RT and Luk JM: MicroRNA-375 targets Hippo-signaling effector YAP in liver cancer and inhibits tumor properties. Biochem Biophys Res Commun 394: 623-627, 2010.

40. Wang Y, Cui M, Sun BD, Liu FB, Zhang XD and Ye LH: miR-506 suppresses proliferation of hepatoma cells through targeting YAP mRNA 3'UTR. Acta Pharmacol Sin 35: 1207-1214, 2014.

41. Bai N, Zhang C, Liang N, Zhang Z, Chang A, Yin J, Li Z, Luo N, Tan X, Luo N, et al: Yes-associated protein (YAP) increases chemosensitivity of hepatocellular carcinoma cells by modulation of p53. Cancer Biol Ther 14: 511-520, 2013.

42. Zhang W and Cohen SM: The Hippo pathway acts via p53 and microRNAs to control proliferation and proapoptotic gene expression during tissue growth. Biol Open 2: 822-828, 2013.

43. Shimizu D, Inokawa Y, Sonohara F, Inaoka K and Nomoto S: Search for useful biomarkers in hepatocellular carcinoma, tumor factors and background liver factors (Review). Oncol Rep 37: 2527-2542, 2017.

44. Noubissi FK, Elcheva I, Bhatia N, Shakoori A, Ougolkov A, Liu J, Minamoto T, Ross J, Fuchs SY and Spiegelman VS: CRD-BP mediates stabilization of betaTrCP1 and c-myc mRNA in response to beta-catenin signalling. Nature 441: 898-901, 2006.

45. Thompson MD and Monga SP: Wnt/beta-catenin signaling in liver health and disease. Hepatology 45: 1298-1305, 2007.
46. Qiao Y, Zhang Y, Wang J: Ubiquitin E3 ligase $\mathrm{SCF}(\beta$-TRCP) regulates TRIB2 stability in liver cancer cells. Biochem Biophys Res Commun 441: 555-559, 2013.

47. Xiao W, Wang J, Ou C, Zhang Y, Ma L, Weng W, Pan Q and Sun F: Mutual interaction between YAP and c-Myc is critical for carcinogenesis in liver cancer. Biochem Biophys Res Commun 439: 167-172, 2013.

48. Xu Y, Stamenkovic I and Yu Q: CD44 attenuates activation of the Hippo signaling pathway and is a prime therapeutic target for glioblastoma. Cancer Res 70: 2455-2464, 2010.

49. Fernandez LA, Northcott PA, Dalton J, Fraga C, Ellison D, Angers S, Taylor MD and Kenney AM: YAP1 is amplified and upregulated in hedgehog-associated medulloblastomas and mediates Sonic hedgehog-driven neural precursor proliferation. Genes Dev 23: 2729-2741, 2009.

50. Yu JZ, Poulton J, Huang YC and Deng WM: The hippo pathway promotes Notch signaling in regulation of cell differentiation, proliferation, and oocyte polarity. PLoS One 3: e1761, 2008.

51. Kim D, Shu S, Coppola MD, Kaneko S, Yuan ZQ and Cheng JQ: Regulation of proapoptotic mammalian ste20-like kinase MST2 by the IGF1-Akt pathway. PLoS One 5: e9616, 2010.

52. Yuan Z, Kim D, Shu S, Wu J, Guo J, Xiao L, Kaneko S, Coppola D and Cheng JQ: Phosphoinositide 3-kinase/Akt inhibits MST1-mediated pro-apoptotic signaling through phosphorylation of threonine 120. J Biol Chem 285: 3815-3824, 2010

53. Joliat GR, Allemann P, Labgaa I, Demartines N and Halkic N Treatment and outcomes of recurrent hepatocellular carcinomas. Langenbecks Arch Surg: May 11, 2017 (Epub ahead of print)

54. Tu K, Yang W, Li C, Zheng X, Lu Z, Guo C, Yao Y and Liu Q Fbxw7 is an independent prognostic marker and induces apoptosis and growth arrest by regulating YAP abundance in hepatocellular carcinoma. Mol Cancer 13: 110, 2014. 